\title{
Mumps (Parotitis epidemica)
}

Aktualisierte Fassung vom Oktober 2013; Erstveröffentlichung im Epidemiologischen Bulletin 37/2001.

RKI-Ratgeber für Ärzte

Herausgeber: Robert Koch-Institut, 2013

Die Herausgabe dieser Reihe durch das Robert Koch-Institut (RKI) erfolgt auf der Grundlage des $\S 4$ Infektionsschutzgesetz (IfSG). Praktisch bedeutsame Angaben zu wichtigen Infektionskrankheiten sollen aktuell und konzentriert der Orientierung dienen. Die Beiträge werden in Zusammenarbeit mit den Nationalen Referenzzentren, Konsiliarlaboratorien sowie weiteren Experten erarbeitet. Die Erstpublikation erfolgt im Epidemiologischen Bulletin und die Publikation von Aktualisierungen im Internet (http://www.rki.de). Eine Aktualisierung erfolgt nach den Erfordernissen, aktualisierte Fassungen ersetzen die älteren. 


\section{Mumps (Parotitis epidemica)}

- Erreger

- Vorkommen

- Reservoir

- Infektionsweg

- Inkubationszeit

- Dauer der Ansteckungsfähigkeit

- Klinische Symptomatik

- Diagnostik

- Therapie

- Präventiv- und Bekämpfungsmaßnahmen

- 1. Präventive Maßnahmen

- 2. Maßnahmen für Patienten und Kontaktpersonen

- Meldepflicht

- Beratung und Spezialdiagnostik

- Ausgewählte Informationsquellen 


\section{Erreger}

Das Mumpsvirus ist ein behülltes einzelsträngiges Negativstrang-RNA-Virus aus der Familie der Paramyxoviridae im Genus Rubulavirus. Die RNA ist von einem Nukleokapsid umgeben; ein Matrixprotein kleidet die Innenseite der Virushülle aus. Die Glykoproteine Hämagglutinin-Neuraminidase und das Fusionsprotein bilden „Spikes“, welche aus der Virushülle herausragen. Das Mumpsvirus ist sehr empfindlich gegenüber äußeren Einflüssen wie Hitze, Licht, UV-Strahlen, fettlösenden Substanzen und Desinfektionsmitteln.

Von Mumpsviren ist nur ein humanpathogener Serotyp bekannt. Mumpsviren werden nach einer WHO-unterstützen Nomenklatur von 2012 in die Genotypen A bis $\mathbf{N}$ eingeteilt und können sich in ihren biologischen Eigenschaften wie der Neurovirulenz unterscheiden. Auch die Fähigkeit zur Kreuzreaktion kann von Genotyp zu Genotyp variieren und unterschiedlich stark ausgeprägt sein. Die einzelnen Genotypen sind geografisch unterschiedlich verbreitet: Die Genotypen A, $C, D, G$ und $H$ werden vor allem in der westlichen Hemisphäre beobachtet, während die Genotypen B, F, I, J/K und L vor allem im asiatischen und pazifischen Raum auftreten. In Deutschland sind Mumps-Erkrankungen in den letzten Jahren vor allem durch den Genotyp G verursacht worden, dessen Auftreten weltweit zunimmt.

\section{Vorkommen}

Mumpsvirus-Infektionen sind weltweit endemisch verbreitet. Sie treten ganzjährig auf, in gemäßigten Klimazonen wurde vor Einführung der Impfung eine Saisonalität mit Häufungen im Winter und Frühjahr beobachtet. In der Vorimpfära bis Mitte der 70er Jahre lag das Prädilektionsalter bei 5- bis 9-jährigen Kindern, die jährliche Inzidenz betrug mehr als 200 Erkrankungen pro 100.000 Einwohner.

Nach dem im Jahr 2001 in Kraft getretenen Infektionsschutzgesetz bestand bis Anfang 2013 in Deutschland für MumpsErkrankungen keine bundesweite Meldepflicht. Ausnahmen hiervon bildeten die fünf östlichen Bundesländer, welche bereits seit 2001 in ihren jeweiligen Landesverordnungen eine Mumps-Meldepflicht für Labore und Ärzte verankert hatten. Aktuelle Daten zur Mumps-Epidemiologie in Deutschland basieren daher auf Mumps-Erfassungen aus den fünf östlichen Bundesländern sowie bundesweit auf Berichten über aufgetretene MumpsErkrankungen in Gemeinschaftseinrichtungen, die nach § 34 IfSG benachrichtigungspflichtig sind.

Mumps-Erkrankungen in den östlichen und westlichen Bundesländern zeigen hinsichtlich der betroffenen Altersgruppen ein unterschiedliches regionales Verteilungsmuster, welches sich durch die verschiedenen Zeitpunkte der Einführung der Mumps-Impfung in West und Ost sowie nachfolgend unterschiedliche Impfquoten erklären lässt (s. Abschnitt „Präventiv- und Bekämpfungsmaßnahmen“).

In den östlichen Bundesländern zeigte sich im Zeitraum von 2001 bis 2012 ein deutlich rückläufiger Trend der Fallzahlen von mehr als 160 Fällen im Jahr 2001 auf 40 Fälle im Jahr 2012. Ab dem Jahr 2002 wurden fortlaufend Inzidenzen unter 1 Erkrankung pro 100.000 Einwohner gemessen. Zwischen 2001 und 2012 wurden insgesamt 858 Mumps-Fälle über das elektronische Meldesystem an das RKI 
übermittelt; davon waren $47 \%$ männlich.

Am häufigsten waren Kinder im Alter unter 15 Jahren betroffen, die

Erkrankungshäufigkeit in dieser Altersgruppe betrug 20 bis 45 Erkrankungen pro 100.000 Kinder. In den Altersgruppen unter 10 Jahre und über 60 Jahre waren Jungen bzw. Männer häufiger betroffen, in den anderen Altersgruppen Mädchen bzw. Frauen.

Zwischen 2001 und 2011 wurden bundesweit mehrere größere Mumps-Ausbrüche registriert. Der größte hiervon war ein überregionaler Ausbruch in Bayern 2010/2011 mit fast 300 übermittelten Fällen. Im Gegensatz zu den Mumps-Geschehen in den östlichen Bundesländern waren die Erkrankten hier älter. Mehr als die Hälfte der Erkrankten waren 16 bis 24 Jahre alt. Es waren vor allem Jugendliche und junge Erwachsene in weiterführenden Schulen, Fachhochschulen, Universitäten und Sportvereinen betroffen.

Daten zur Mumps-Epidemiologie wie diese aus dem bayerischen Ausbruch geben Hinweise, dass eine Rechtsverschiebung des Erkrankungsalters in den westlichen Bundesländern bereits stattgefunden hat. Dies ist von besonderem Interesse, da mit steigendem Erkrankungsalter eine Zunahme der Komplikationsrate im Rahmen der Mumps-Erkrankung beobachtet wird.

Die in den Ausbrüchen beobachtete Altersverschiebung in den westlichen Bundesländern wurde auch in anderen Ländern Europas registriert: SurveillanceDaten anderer europäischer Länder wie z. B. aus Großbritannien, Irland, Spanien und Tschechien lassen erkennen, dass das Erkrankungsalter heutzutage im Durchschnitt deutlich höher ist und ein Großteil der symptomatischen Erkrankungen Jugendliche und junge Erwachsene betrifft. Die höchsten Inzidenzen wurden dabei bei den 15- bis 19-Jährigen beschrieben.

Lang anhaltende und umfangreiche Ausbrüche wurden in den letzten 10 Jahren auch in den USA und Kanada beobachtet: Auch hier waren meist Jugendliche und junge Erwachsene in Schulen und Universitäten betroffen. Viele der Erkrankten hatten eine abgeschlossene Grundimmunisierung. Der größte Ausbruch in den USA mit über 6.500 Fällen betraf vornehmlich College- und Universitätsstudenten, von denen über $60 \%$ zwei Impfungen erhalten hatten.

Als Ursache für die Altersverschiebung und die Infektionen unter zweifach Geimpften werden vor allem ein über die Zeit abnehmender Impfschutz (Waning immunity), eine mangelnde Boosterung durch weniger zirkulierende Wildviren aufgrund von steigenden Impfquoten unter Kindern sowie mögliche Antigenunterschiede zwischen Impfstoffvirus und Ausbruchsstamm („AntigenMismatch") diskutiert. Neben diesen Gründen sind für die Altersverschiebung in Deutschland sicher auch die mangelhaften Impfquoten für die zweifache MumpsImpfung verantwortlich, die bis 2001 bei den Schuleingangsuntersuchungen unter $50 \%$ betrugen. 


\section{Reservoir}

Der Mensch ist das einzige Erregerreservoir.

\section{Infektionsweg}

Die Übertragung erfolgt vor allem aerogen durch Tröpfcheninfektion und direkten Speichelkontakt, seltener durch mit Speichel kontaminierte Gegenstände. Die mögliche Virusausscheidung im Urin und in der Muttermilch hat meist keine praktische Bedeutung für die Übertragung.

\section{Inkubationszeit}

Die Inkubationszeit beträgt in der Regel 16 bis 18 Tage (12 bis 25 Tage sind möglich).

\section{Dauer der Ansteckungsfähigkeit}

Die Ansteckungsfähigkeit ist 2 Tage vor bis 4 Tage nach Erkrankungsbeginn am größten. Insgesamt kann ein Infizierter 7 Tage vor bis 9 Tage nach Auftreten der Parotisschwellung ansteckend sein. Auch klinisch inapparente Infektionen sind ansteckend.

\section{Klinische Symptomatik}

Mumps (Parotitis epidemica) ist eine systemische Infektionskrankheit, die in jedem Lebensalter auftreten kann. Sie führt in der Regel zu lebenslanger Immunität; Reinfektionen sind möglich, aber selten. Zusätzlich ist zu beachten, dass eine vollständige Grundimmuniserung mit zwei Impfungen eine Mumps-Erkrankung nicht vollständig ausschließt.

Typischerweise ist die Mumps-Erkrankung durch eine schmerzhafte einseitige (20 $30 \%)$ bzw. doppelseitige (70-80 \%) entzündliche Schwellung der Parotis gekennzeichnet, welche etwa 3 bis 8 Tage andauert. Bei $10-15 \%$ der Krankheitsfälle wird eine Beteiligung der submandibulären bzw. der sublingualen Speicheldrüsen beobachtet. Mögliche Differenzialdiagnosen sind unter anderem virale Infektionen mit dem Epstein-Barr-Virus, Parainfluenzavirus (Typ 1 und 3), Influenzavirus A, Coxsackievirus, Adenovirus und Parvovirus B19. Vorausgehen kann der Infektion ein mehrtägiges Prodromalstadium mit Fieber, Kopfschmerz, Unwohlsein, Myalgien und Appetitverlust. Der Großteil der Mumps-Infektionen im Alter unter 2 Jahren verläuft subklinisch, jedoch präsentiert sich die Mumps-Infektion bei Kindern unter 5 Jahren häufig als akute respiratorische Erkrankung (40-50\% der Fälle). Mindestens 30-40\% der Infektionen verlaufen klinisch inapparent oder subklinisch. 
Im Rahmen der Erkrankung kann eine Reihe von Komplikationen auftreten, die mit steigendem Alter häufiger werden. Eine ZNS-Beteiligung, von der Männer häufiger betroffen sind als Frauen, gehört bei Mumps-Infektionen mit ca. 60 \% zu den häufigsten Komplikationen. Sie reicht von einer asymptomatischen Pleozytose bei bis zu $50 \%$ der Fälle bis zur Enzephalitis bei unter $1 \%$ der Mumps-Fälle. Eine MumpsEnzephalitis verläuft bei 1,5\% der Fälle tödlich. Bei 1-10\% der Fälle werden symptomatische aseptische Meningitiden beobachtet, die jedoch nicht mit persistierenden Spätfolgen oder letalem Ausgang assoziiert sind. Eine transiente Taubheit im Hochfrequenzbereich wurde bei ca. $4 \%$ der Mumps-Fälle beobachtet, eine persistierende unilaterale Taubheit tritt bei 1 von 20.000 Mumps-Fällen auf. ZNS-Beteiligungen manifestieren sich meist 4 bis 5 Tage nach der Parotitis und dauern 7 bis 10 Tage an; sie können der Parotitis aber auch vorangehen oder einziges Symptom der Mumps-Erkrankung sein.

Eine häufigere Komplikation ist mit $15-30 \%$ die Orchitis (15-30\% davon bilateral) beim erwachsenen Mann, welche sich normalerweise 4 bis 8 Tage nach der Parotitis manifestiert und für etwa 1 bis 2 Wochen fortbesteht. Eine testikuläre Atrophie wird in $40-70 \%$, ein abnormes Spermatogramm in bis zu $25 \%$ der Orchitisfälle beschrieben, eine resultierende Sterilität ist jedoch selten. Bei der erwachsenen Frau kann im Rahmen der Mumps-Infektion bei bis zu $30 \%$ der Fälle eine Mastitis und bei bis zu $5 \%$ eine Oophoritis auftreten. Eine Mumps-Erkrankung während der Schwangerschaft ist nicht mit einer erhöhten Rate an kongenitalen Missbildungen assoziiert. Hinweise auf eine erhöhte Abortrate infolge einer Mumps-Infektion während des ersten Trimesters wurden bislang nicht bestätigt.

Als Komplikationen der Mumps-Infektion können zusätzlich eine Pankreatitis (ca. 4 \% der Fälle), Nephritis, Arthritis, Anämie oder Myokarditis mit EKGVeränderungen auftreten

\section{Diagnostik}

Bei einem typischen Verlauf von Mumps kann die Diagnose zwar häufig klinisch anhand der charakteristischen Ohrspeicheldrüsenschwellung gestellt werden, jedoch sollte zur eindeutigen Sicherung bei den heutzutage niedrigen Mumps-Inzidenzen eine Laboruntersuchung veranlasst werden. Insbesondere beim Nachweis von Erkrankungen bei Geimpften ist die Labordiagnostik (Serologie und PCR, s. unten) für den Nachweis der Mumps-Erkrankung unerlässlich.

Für die Labordiagnostik steht ein breites Spektrum von Methoden zur Verfügung, die den Nachweis spezifischer Antikörper und den Virusnachweis umfassen:

Die akute Mumps-Erkrankung kann mittels ELISA über den Nachweis von virusspezifischen IgM-Antikörpern labordiagnostisch gesichert werden. IgMAntikörper sind bereits in den ersten Tagen der Erkrankung nachweisbar, erreichen ihr Maximum etwa 7 Tage nach Symptombeginn und bleiben über Wochen erhöht.

Bei Personen, die eine Mumps-Erkrankung entwickeln, obwohl sie geimpft sind, ist IgM häufig nicht nachweisbar. Mumps kann bei dieser Gruppe daher auf Basis eines negativen IgM-Nachweises nicht sicher ausgeschlossen werden. In diesen Fällen sollte möglichst eine weitere Serumprobe im Abstand von 10 bis 14 Tagen 
untersucht werden. Im Vergleich der beiden Serumproben-Titer kann dann ggf. ein signifikanter IgG-Antikörperanstieg nachgewiesen werden. Da ein ausbleibender Titer-Anstieg Mumps bei Geimpften jedoch ebenfalls nicht sicher ausschließt, sollte bei geimpften Patienten neben der Serologie unbedingt eine RT-PCR aus Urin, Rachenabstrich oder Zahntaschenflüssigkeit (oral fluid) veranlasst werden.

Die Proben für die PCR, welche kostenfrei im Nationalen Referenzzentrum (NRZ) für Masern, Mumps, Röteln durchgeführt wird (s. Abschnitt „Beratung und Spezialdiagnostik"), sollten möglichst umgehend nach Symptombeginn abgenommen werden. Eine PCR kann auch bei besonderen Fällen, z. B. ZNS-Manifestationen, aus Liquor oder Biopsiematerial durchgeführt werden. Im Falle eines positiven RNANachweises kann im Nationalen Referenzzentrum für Masern, Mumps, Röteln eine MumpsGenotypisierung erfolgen.

\section{Therapie}

Die Therapie ist ausschließlich symptomatisch (z. B. Analgetika, Antipyretika). Eine spezifische antivirale Therapie gegen Mumps gibt es nicht.

\section{Präventiv- und Bekämpfungsmaßnahmen}

\section{Präventive Maßnahmen}

Die wirksamste präventive Maßnahme ist die Schutzimpfung gegen Mumps. Dabei ist bekannt, dass die zweite Impfung den Impfschutz verbessert. Der MumpsImpfstoff ist ein Lebendimpfstoff und enthält attenuierte, auf Hühnerembryozellen gezüchtete Mumpsviren (Stamm Jeryl Lynn bzw. davon abgeleitet RIT 4385); er ist seit dem Jahr 1976 als Masern-Mumps-Röteln- bzw. seit 2006 als Masern-MumpsRöteln-Varizellen-Kombinationsimpfstoff (MMR- bzw. MMRV-Vakzine) zugelassen. Die Impfung erzeugt sowohl eine humorale als auch zellulär vermittelte Immunität. Eine Altersbegrenzung für die Gabe der Impfung existiert nicht.

1976 wurde erstmalig von der Ständigen Impfkommission (STIKO) in der Bundesrepublik eine Empfehlung für eine einmalige Mumps-Impfung (ab dem 2. Lebensjahr) ausgesprochen; in der DDR wurde nicht gegen Mumps geimpft. Ab 1991 empfahl die STIKO gesamtdeutsch die Gabe von zwei Impfdosen (2. Lebensjahr und 6. Lebensjahr) - vorzugsweise mit MMR-Kombinationsimpfstoff. Seit 2001 gilt die aktuell bestehende Impfempfehlung, die 1. Dosis im Alter von 11 bis 14 Monaten, gefolgt von der 2. Dosis im Alter von 15 bis 23 Monaten zu verabreichen.

Im Rahmen der jährlich durchgeführten Schuleingangsuntersuchungen zeigt sich, dass die Mumps-Impfquoten in den letzten 10 Jahren kontinuierlich gestiegen sind. Zum Schuleingang im Jahr 2000 lag die Impfquote für die erste Mumps-Impfung bei $90,8 \%, 2005$ bei $93,7 \%$ und 2010 bei $96,1 \%$. Eine zweite Mumps-Impfung hatten im Jahr 2000 19,0 \% erhalten, 2005 waren es 76,4 \% und im Jahr 2010 91,2\%. Die Impfquoten sind in den östlichen Bundesländern sowohl für die erste Impfung (2010: $97,6 \%$ vs. $95,8 \%$ ) wie für die zweite Impfung (2010: $92,7 \%$ vs. $91,0 \%$ ) höher als in den westlichen Bundesländern. Diese Impqquoten, die auf der Basis der Einträge in 
den vorgelegten Impfausweisen berechnet werden, stellen vermutlich eine leichte Überschätzung dar, da Kinder ohne Impfdokumente in der Regel schlechter geimpft sind.

Um Ausbrüche oder eine kontinuierliche Zirkulation des Mumpsvirus zu vermeiden, sollte bundesweit eine Mumps-Impfquote in der Bevölkerung von mindestens $95 \%$ für die zweite Impfung erreicht werden. Zusätzlich sollten Immunitätslücken bei Jugendlichen geschlossen werden.

Berufliche Impfindikationen bestehen für nach 1970 Geborene mit unklarem Impfstatus, ohne Impfung oder nur einer Impfung in der Kindheit, die in Gesundheitsdienstberufen in der unmittelbarem Patientenversorgung, in Gemeinschaftseinrichtungen oder Ausbildungseinrichtungen für junge Erwachsene tätig sind.

\section{Maßnahmen für Patienten und Kontaktpersonen}

\section{Maßnahmen bei Erkrankten}

An Mumps erkrankte Patienten sollten bei stationärer Behandlung von anderen Patienten getrennt untergebracht werden. Nach § 34 des Infektionsschutzgesetzes dürfen Personen, die an Mumps erkrankt oder dessen verdächtig sind, solange in Gemeinschaftseinrichtungen keine Lehr-, Erziehungs-, Pflege-, Aufsichts- oder sonstigen Tätigkeiten (bei denen sie Kontakt zu den dort Betreuten haben) ausüben, bis nach ärztlichem Urteil eine Weiterverbreitung der Krankheit durch sie nicht mehr zu befürchten ist.

Entsprechend dürfen auch die in Gemeinschaftseinrichtungen Betreuten, die an Mumps erkrankt oder dessen verdächtig sind, weder dem Betrieb der Gemeinschaftseinrichtung dienende Räume betreten, Einrichtungen der Gemeinschaftseinrichtung nutzen noch an Veranstaltungen der Gemeinschaftseinrichtung teilnehmen.

Eine Wiederzulassung zu Gemeinschaftseinrichtungen kann nach Abklingen der klinischen Symptome, jedoch frühestens 5 Tage nach dem Beginn der MumpsErkrankung erfolgen. Ein schriftliches ärztliches Attest ist nicht erforderlich.

\section{Maßnahmen bei Kontaktpersonen}

Personen, in deren Wohngemeinschaft nach ärztlichem Urteil eine Mumps-

Erkrankung oder ein Verdacht auf eine Mumps-Erkrankung aufgetreten ist und die als ansteckungsverdächtig anzusehen sind, dürfen solange in

Gemeinschaftseinrichtungen keine Tätigkeiten ausüben, bei denen sie Kontakt zu Betreuten haben bzw. diese als Betreute/r besuchen, bis nach ärztlichem Urteil eine Weiterverbreitung der Krankheit durch sie nicht mehr zu befürchten ist (IfSG $\S 34$ Abs. 3).

Empfehlungen zum Kontaktmanagement in Abhängigkeit vom Impf- und Immunstatus sind in Tabelle 1 detailliert dargestellt. 


\begin{tabular}{|c|c|c|}
\hline Immunstatus & $\begin{array}{l}\text { Zeitdauer seit } \\
\text { erstem Kontakt mit } \\
\text { Mumps-Fall }\end{array}$ & Maßnahme/Empfehlung \\
\hline $\begin{array}{l}2 \text { dokumentierte } \\
\text { Impfungen }\end{array}$ & nicht relevant & $\begin{array}{l}\text { keine Maßnahmen } \\
\text { kein Ausschluss }\end{array}$ \\
\hline $\begin{array}{l}1 \text { dokumentierte } \\
\text { Impfung }\end{array}$ & nicht relevant & $\begin{array}{l}\text { - Ausschluss } \\
\text { - } \text { Wiederzung: 2. Impfung } \\
\text { nachgewiesener 2. Impfung } \\
\text { (jedoch unabhängig vom Zeitpunkt der } \\
\text { 2. Impfung) }\end{array}$ \\
\hline ungeimpft & > 3 Tage & $\begin{array}{l}\text { - } \quad \text { Ausschluss für Dauer der mittleren } \\
\text { Inkubationszeit (18 Tage) } \\
\text { - Impfempfehlung (erfolgte Impfung führt } \\
\text { aber nicht zur Wiederzulassung) }\end{array}$ \\
\hline & $\leq 3$ Tage & $\begin{array}{l}\text { - Ausschluss } \\
\text { - Empfehlung: sofortige Impfung } \\
\text { niederzulassung erst nach } \\
\text { nachgewiesener einmaliger Impfung }\end{array}$ \\
\hline $\begin{array}{l}\text { keine dokumentierte } \\
\text { Impfung } \\
\text { oder } \\
\text { unklare Mumps- } \\
\text { Anamnese }\end{array}$ & $>3$ Tage & $\begin{array}{l}\text { - Ausschluss } \\
\text { - } \text { Mumps-Antikörper-Bestimmung } \\
\text { Empfehlung: sofortige Impfung nach } \\
\text { - Wientenahme } \\
\text { positivem Mumpung-lgG-Antikörpertiter } \\
\text { (bei negativem Befund Ausschluss für } \\
\text { die Dauer der mittleren Inkubationszeit } \\
\text { (18 Tage)) }\end{array}$ \\
\hline & $\leq 3$ Tage & $\begin{array}{l}\text { - Ausschluss } \\
\text { - Empfehlung: sofortige Impfung } \\
\text { nacderzulassung erst nach } \\
\text { nachgewiesener einmaliger Impfung }\end{array}$ \\
\hline $\begin{array}{l}\text { Impfbuch nicht } \\
\text { vorgelegt }\end{array}$ & nicht relevant & $\begin{array}{l}\text { - Ausschluss } \\
\text { - Wiederzulassung erst nach } \\
\text { Impfbuchkontrolle (je nach Ergebnis, } \\
\text { s. o.) } \\
\text { bzw. positivem Mumps-lgG- } \\
\text { Antikörpertiter }\end{array}$ \\
\hline $\begin{array}{l}\text { glaubwürdige (z. B. } \\
\text { vor } 1970 \text { Geborene) } \\
\text { oder } \\
\text { dokumentierte } \\
\text { frühere Mumps- } \\
\text { Erkrankung }\end{array}$ & nicht relevant & $\begin{array}{l}\text { keine Maßnahmen } \\
\text { kein Ausschluss }\end{array}$ \\
\hline
\end{tabular}


Tab. 1: Empfohlene Maßnahmen zum Kontaktmanagement von Personen nach Mumpskontakt, die Gemeinschaftseinrichtungen besuchen oder in ihnen tätig sind (aus: Epid. Bull. 2012; 22: 205-211)

\section{Maßnahmen bei Ausbrüchen}

Mumps-Fälle in Gemeinschaftseinrichtungen müssen dem zuständigen Gesundheitsamt unverzüglich durch die Leitung der Einrichtung gemäß IfSG § 34 Abs. 6 gemeldet werden. Das Gesundheitsamt hat so zeitnah die Möglichkeit, Maßnahmen zur Verhütung weiterer Erkrankungen einzuleiten.

Wichtigste Maßnahme zur Verhinderung einer weiteren Ausbreitung ist die postexpositionelle Impfung bisher ungeimpfter bzw. nur einmal geimpfter Kontaktpersonen (Riegelungsimpfung), die möglichst innerhalb der ersten 3 Tage nach Exposition erfolgen sollte. Für die Wiederzulassung in Gemeinschaftseinrichtungen gelten die unter Punkt 2 beschriebenen Empfehlungen für Maßnahmen bei Kontaktpersonen.

\section{Meldepflicht}

Seit dem 29.3.2013 besteht eine Mumps-Meldepflicht gemäß IfSG.

Gemäß § 6 IfSG sind der Krankheitsverdacht, die Erkrankung sowie der Tod an Mumps namentlich an das zuständige Gesundheitsamt zu melden. Gemäß § 7 IfSG besteht eine Meldepflicht für den direkten oder indirekten Nachweis einer akuten Mumpsvirus-Infektion.

Weiterhin hat gemäß § 34 Abs. 6 IfSG die Leitung einer Gemeinschaftseinrichtung das zuständige Gesundheitsamt unverzüglich über das ihr zur Kenntnis gelangte Auftreten von Mumps-Erkrankungen in der Einrichtung zu benachrichtigen und dazu krankheits- und personenbezogene Angaben zu machen.

Falldefinitionen für Gesundheitsämter: Die vom RKI erarbeitete Falldefinition für Mumps wird im Internet unter www.rki.de > Infektionsschutz > Infektionsschutzgesetz $>$ Falldefinitionen und im Bundesgesundheitsblatt veröffentlicht.

\section{Beratung und Spezialdiagnostik}

\section{Beratung zur Epidemiologie und zur Impfempfehlung}

Abteilung für Infektionsepidemiologie

Fachgebiet Impfprävention

Robert Koch-Institut

Seestraße 10, 13353 Berlin

Dr. Judith Koch, Dr. Anja Takla

Tel.: + $49(0) 30.18754-3484,-3483$

Fax: $+49(0) 30.18754-3533$

E-Mail: Dr. Judith Koch; Dr. Anja Takla 


\section{Beratung zur Spezialdiagnostik}

Nationales Referenzzentrum für Masern, Mumps, Röteln

Robert Koch-Institut

Seestraße 10, 13353 Berlin

Leitung: PD Dr. Annette Mankertz

Tel.: $+49(0) 30.18754-2516,-2308$

Fax: $+49(0) 30.18754-2598$

E-Mail: Dr. Annette Mankertz

\section{Ausgewäh/te Informationsquellen}

1. WHO: Mumps virus nomenclature update: 2012. Wkly Epidemiol Rec 2012; 87(22): 217-224

2. Anis E, Grotto I, Moerman L, Warshavsky B, Slater PE, Lev B: Mumps outbreaks in Israel's highly vaccinated society: are two doses enough? Epidemiol Infect 2011; doi:10.1017/S095026881100063X

3. Davidkin I, Kontio M, Paunio M, Peltola $H$ : MMR vaccination and disease elimination: the Finnish experience. Expert review of vaccines 2010;9(9): 1045-1053

4. Dayan GH, Quinlisk MP, Parker AA et al.: Recent Resurgence of Mumps in the United States. N Engl J Med 2008; 358: 1580-1589

5. Dayan GH, Rubin S: Mumps Outbreaks in Vaccinated Populations: Are Available Mumps Vaccines Effective Enough to Prevent Outbreaks? CID 2008; 47: 1458-1467

6. Demicheli V, Rivetti A, Debalini MG, Di Pietrantonj C: Vaccines for measles, mumps and rubella in children. Cochrane database of systematic reviews 2012; 2: CD004407. Epub 2012/02/18

7. ECDC: Mumps Surveillance Annual Report 2010. Erhältlich unter: http://ecdc.europa.eu/en/publications/Publications/mumps report 2010 euvac net.pdf http://www.euvac.net/graphics/euvac/pdf/mumps report 2009.pdf. Zugegriffen am 9. Mai 2012

8. Enders M, Rist B, Enders G: Abort- und Frühgeburtenrate nach akuter Mumpsinfektion in der Schwangerschaft. Gynäkol Geburtshilfliche Rundsch 2005; 45: 39-43

9. Eriksen J, Davidkin I, Kafatos G, Andrews N, Barbara C, Cohen D, et al.: Seroepidemiology of mumps in Europe (1996 - 2008): why do outbreaks occur in highly vaccinated populations? Epidemiol Infect 2012: 1-16. Epub 2012/06/13

10. Galazka, AM, Robertson SE, A Kraigher: Mumps and mumps vaccine: a global review. Bull World Health Organ 1999; 77(1): 3-14

11. Heininger $\mathrm{U}$, Bonhoeffer $\mathrm{J}$ : Interstrain antigenic variability of mumps viruses. CID 2008; 46:150-151

12. Heymann D L (ed): Control of Communicable Diseases Manual. American Public Health Association; 2008, 431-434

13. Hviid A, Rubin S, Mühlemann K: Mumps. Lancet 2008; 371: 932-944

14. Kutty PK, Kruszon-Moran DM, Dayan GH, et al.: Seroprevalence of antibody to mumps virus in the US population, 1999-2004. J Infect Dis 2010; 202: 667 
15. Kutty PK, Kyaw MH, Dayan GH et al.: Guidance for Isolation Precautions for Mumps in the United States: A Review of the Scientific Basis for Policy Change. CID 2010; 50: 1619-1628

16. Leineweber B, Grote V, Schaad UB, Heininger U: Transplacentally acquired immunoglobulin $\mathrm{G}$ antibodies against measles, mumps, rubella and varicellazoster virus in preterm and full term newborns. Pediatr Infect Dis J 2004; 23(4): 361-363

17. Levine H, Rishpon S, Huerta-Hartal M, Davidovitch N: Preventing mumps outbreaks in confined settings. Human Vaccines 2011; 7: 1389-1393

18. Rubin S, Plotkin SA. Mumps vaccine. In: Plotkin SA, Orenstein WA, Offit PA, editors: Vaccines. 6 ed. Philadelphia PA, Saunders, 2013, 419-446

19.Pöhn HP RG: Statistik meldepflichtiger übertragbarer Krankheiten. München, MMV Medizin Verlag, 1994

20. Quinslisk MP: Mumps Control Today. JID 2010; 202: 655-656

21. Reiter S, Poethko-Muller C: Aktuelle Entwicklung von Impfquoten und Impflücken bei Kindern und Jugendlichen in Deutschland.

Bundesgesundheitsbl - Gesundheitsforsch - Gesundheitsschutz 2009; 52(11): 1037-1044

22. RKI: Impfpräventable Krankheiten bis zum Jahr 2000. Jahresbericht. Epid Bull 2002; 7: 49-57

23. RKI: Empfohlene immunprophylaktische Maßnahmen bei Auftreten von Erkrankungen an Masern, Mumps oder Röteln in Kindereinrichtungen und Schulen. Epid Bull 2001; 29: 222-223

24. RKI: Impfempfehlungen der Ständigen Impfkommission (STIKO) am Robert Koch-Institut/Stand: Juli 2011. Epid Bull 2011; 30: 275-294

25. RKI: Mumpsausbruch an einer Grundschule in Nürnberg 2011. Epid Bull 2012; 22: 205-211

26. RKI: Impfquoten bei den Schuleingangsuntersuchungen in Deutschland 2010. Epid Bull 2012; 16: 135-139

27. Vandermeulen C, Leroux-Roels G, Hoppenbrouwers K: Mumps outbreaks in highly vaccinated populations. What makes good even better? Human Vaccines 2009; 5: 294-296 (2)

28. Wichmann O, Hellenbrand W, Sagebiel D et al.: Large measles outbreak at a German public school, 2006. Pediatr Infect Dis J 2007; 26: 782-786

29. WHO: Mumps virus vaccines. WHO position paper. Wkly Epidemiol Rec 2007; 82(7): 51-60

Hinweise zur Reihe „RKI-Ratgeber für Ärzte“ bitten wir an das RKI, Abteilung für Infektionsepidemiologie (Tel.: +49 (0)30 - 18754-3312, Fax: +49 (0)30 - 18754-3533) oder an die Redaktion des Epidemiologischen Bulletins zu richten.

Stand: 20.12.2013 